\title{
Analysis of Two-way Time Synchronization Error Caused by Satellite Motion
}

\author{
Wei Li ${ }^{1, \text { a }}$, Xiang Wang ${ }^{2, \text { a }}$,Huijie Song ${ }^{2,}$, Jihai Zhang ${ }^{2, \text { a }}$ \\ ${ }^{1}$ National Time Service Center, Chinese Academy of Sciences, Xi'an 710600, China \\ ${ }^{2}$ Key Laboratory of Time \& Frequency Primary Standards, Chinese Academy of Sciences, Xi'an \\ 710600, China \\ aemail:kim_weili@ntsc.ac.cn
}

Keywords: TWSTFT, path symmetry, satellite motion, time synchronization error

\begin{abstract}
In a navigation satellite system, the two-way satellite time and frequency transfer (TWSTFT) is mainly used for time synchronization at earth stations, and system time tracing, etc. The reason why the TWSTFT can realize high-precision time synchronization is its path symmetry; however, in practical applications, satellite motion leads to a deviation from the distance between base station and satellite and a time synchronization error between two base stations, which affect the path symmetry of the TWSTFT and further influence the precision of two-way satellite time synchronization. In this paper, the calculation method of the two-way time synchronization error caused by satellite motion was studied, and the influence of satellite motion on the precision of two-way time synchronization was analyzed for three base stations, i.e., Sanya, Chengdu and Kashi, selected at low, medium and high latitudes, respectively, with Beijing station as a reference station, using the orbital data of different satellites on one day. In the two-way time synchronization technology research for and construction of the BeiDou Navigation Satellite System, if geosynchronous earth orbit (GEO) satellites are used for two-way time transfer with precision of 1 ns, the influence of satellite motion can be neglected; however, if it is expected to realize time comparison with higher precision or to use satellites with faster motion velocity, the research results from this paper will have important reference function.
\end{abstract}

\section{Introduction}

The TWSTFT is a method for comparing time scales and atomic frequency standards with the highest precision and the strongest real-time performance internationally recognized at present [1][2], in which the uncertainty of time comparison is less than $1 \mathrm{~ns}$ [3]. In a navigation satellite system, the two-way satellite time and frequency transfer (TWSTFT) is primarily used to realize time synchronization at earth stations, and system time tracing, etc.

The reason why the TWSTFT can realize high precision time synchronization is its path symmetry[4]; however, in practical applications, satellite motion leads to a deviation from the distance between base station and satellite and a time synchronization error between two base stations, which change the geometric paths between base station and satellite, affect the path symmetry of the TWSTFT and further influence the precision of two-way satellite time synchronization.

If GEO satellites are used for two-way time transfer with precision of $1 \mathrm{~ns}$, the influence of satellite motion can be neglected; however, if it is expected to realize time comparison with higher precision or to use satellites with faster motion velocity, such as IGSO, it is quite necessary to study the influence of satellite motion on the precision of two-way time synchronization. 


\section{Principle of Path Asymmetry Caused By Satellite Motion}

During two-way time transfer, satellite motion may cause asymmetric uplink and downlink signal transmission paths, and further lead to comparison error[5], as shown in Fig 1.

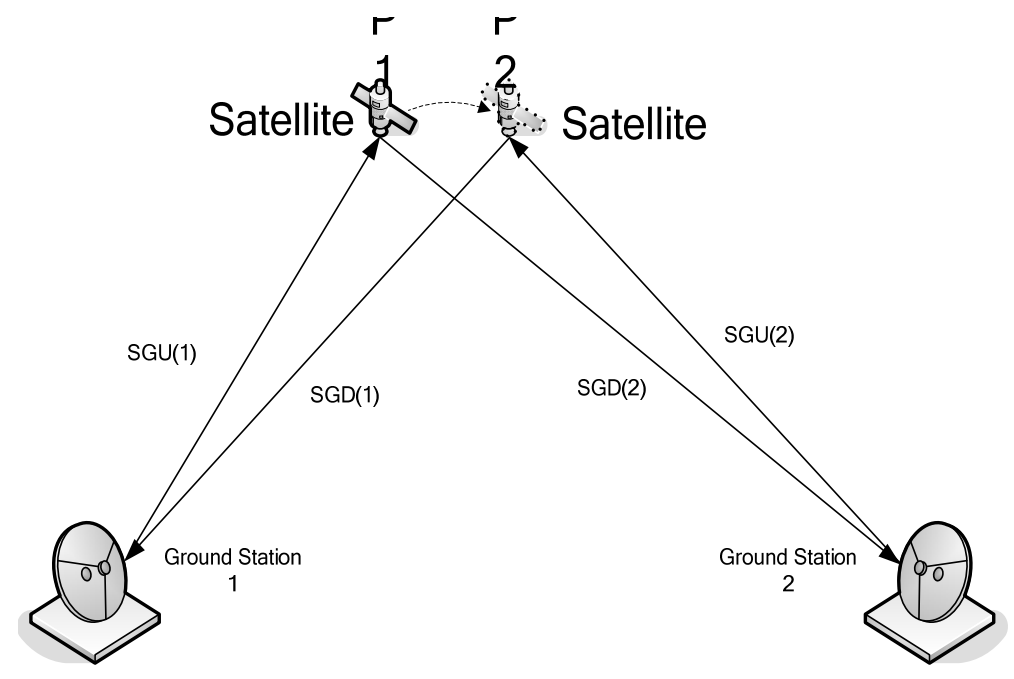

Fig.1.Schematic of path symmetry caused by satellite motion

The signal sent to a satellite by Earth Station1 reaches the satellite at time point $t_{1}$, when the satellite is located at position P1; and the signal sent to the satellite by Earth Station 2 reaches the

satellite at time point $t_{2}$, when the satellite is located at position P2. As can be known from Fig.1, satellite motion leads to the following: $\mathrm{SGU}(1) \neq \mathrm{SGD}(1)$, and $\mathrm{SGU}(2) \neq \mathrm{SGD}(2)$, where SGU is the geometric time delay in signal uplink and SGD is the geometric time delay in signal downlink; the geometric time delays cannot be offset completely through subtraction after data exchange between these two stations. Therefore, the influence of satellite motion in two-way time comparison is:

$$
\begin{aligned}
\tau & =1 / 2\{[\operatorname{SGU}(1)-\operatorname{SGD}(1)]-[\operatorname{SGU}(2)-\mathrm{SGD}(2)]\} \\
& =1 / 2\{[\mathrm{SGU}(1)+\mathrm{SGD}(2)]-[\mathrm{SGU}(2)+\mathrm{SGD}(1)]\}
\end{aligned}
$$

It can be known from the above analysis that the difference between the time delay when Station 1 receives signal and that when Station 2 receives signal is the influence of satellite motion on two-way time synchronization.

\section{Flow of Calculation of Path Asymmetry Caused By Satellite Motion}

The calculation flow is shown in Fig2. 


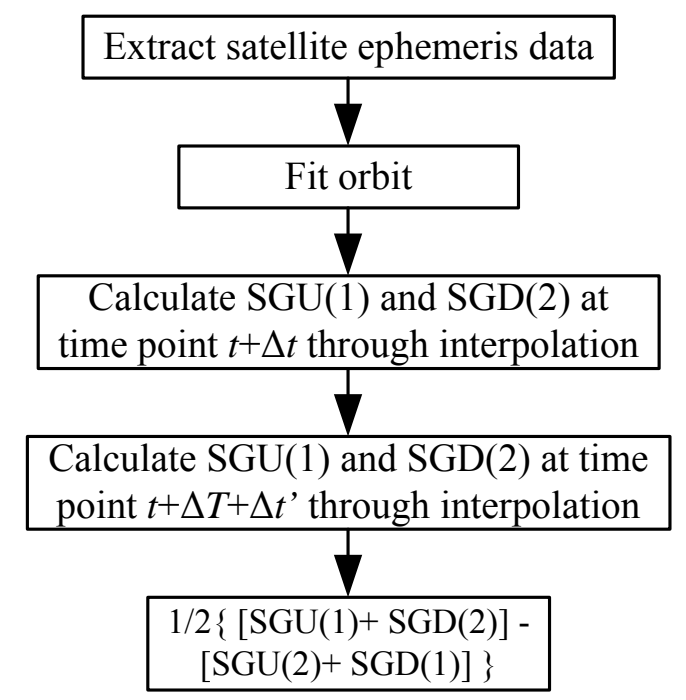

Fig.2.Flow of calculation of path asymmetry caused by satellite motion

For Earth Stations 1 and 2, the ephemeris data (at one minute interval) of a satellite on one day were selected to fit the orbit of the satellite. When the time for the signal sent from Station 1 at time point $\mathrm{t}$ to reach the satellite is $\Delta t$, the time delay from Station 1 to the satellite and that from the satellite to Station 2 at time point $t+\Delta t$ are calculated through interpolation as $\mathrm{SGU}(1)$ and SGD(2), respectively. When the time for the signal sent from Station 2 at time point to reach the satellite is $\Delta t^{\prime}$, and it is assumed that the clock bias between Stations 1 and 2 is $\Delta T$, the time delay from Station 2 to the satellite and that from the satellite to Station 1 at time point $t+\Delta t^{\prime}+\Delta T$ are calculated through interpolation as $\operatorname{SGU}(2)$ and $\operatorname{SGD}(1)$, respectively. The deviation between the time delay for the signal sent from Station 1 to reach Station 2 via the satellite and that for the signal sent from Station 2 to reach Station 1 via the satellite at time point $t$ when the clock bias between these two stations is $\Delta T$ can be obtained from calculation according to Eq. (1).

\section{Experimental Results}

The influence of satellite motion on the precision of two-way time synchronization was analyzed for three base stations, Sanya, Chengdu and Kashi, selected at low, medium and high latitudes in China, respectively, with Beijing station as a reference station, using the orbital data one GEO satellite and IGSO satellite on one day.

SinoSat-1 GEO communication satellite

Fig 3 shows the variation in distance from Beijing to the SinoSat-1 satellite on one day according to the orbital data; 


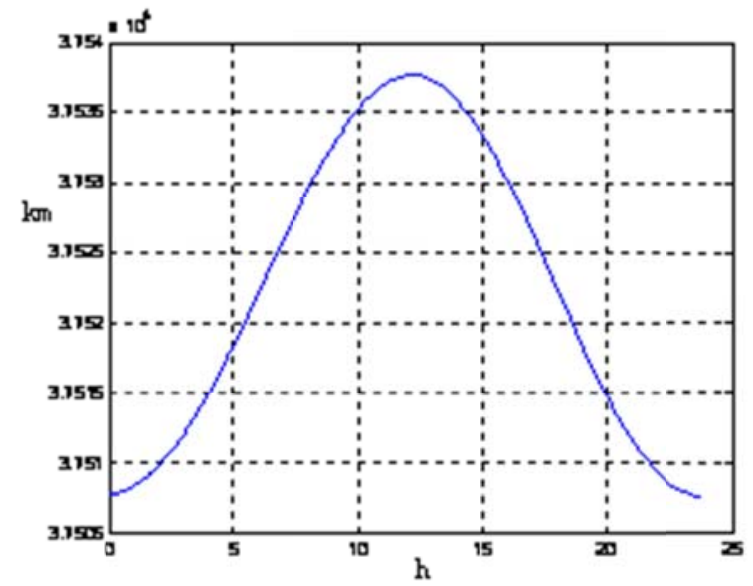

Fig.3.Variation in distance from Beijing to the SinoSat-1 satellite

As can be seen from Fig. 3, the variation in distance from Beijing to the SinoSat-1 satellite within one day exhibits a sine wave trend and its range is not more than $30 \mathrm{~km}$.

Fig 4 shows the variations with time in time synchronization error between the time delay for the signal sent from Beijing Station to reach Chengdu/Kashi/SanyaStation via the SinoSat-1 satellite and that for the signal sent from Chengdu/Kashi/SanyaStation to reach Bieijing Station via the SinoSat-1 satellite when the clock biases between twostations are $1 \mu \mathrm{s}, 10 \mu \mathrm{s}, 100 \mu \mathrm{s}, 1 \mathrm{~ms}$ and $10 \mathrm{~ms}$ respectively.

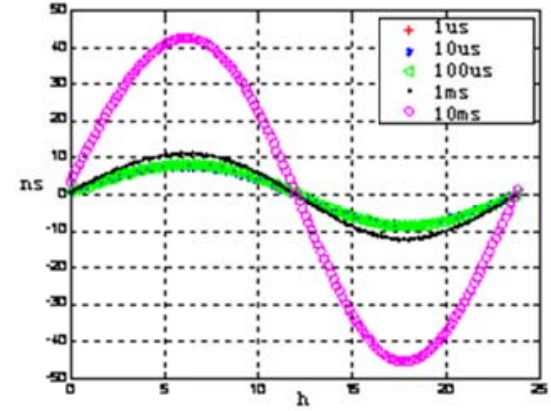

(a) Beijing-Chengdu
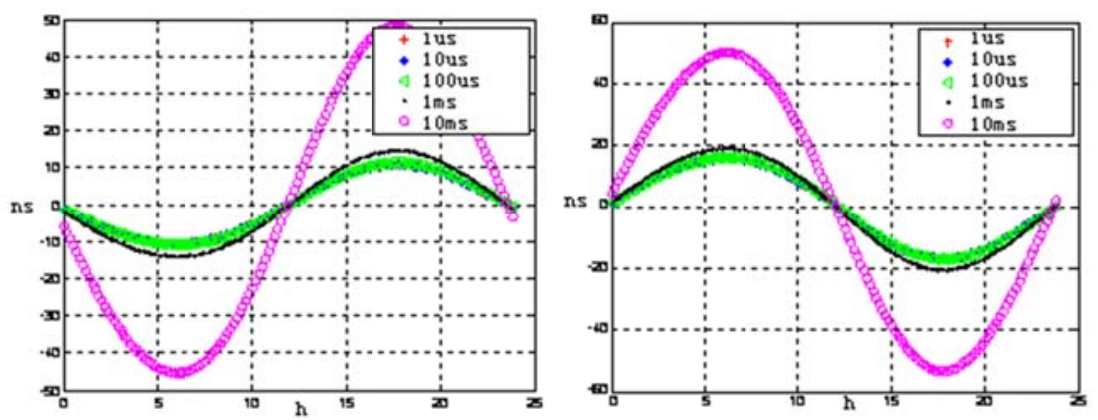

(b)Beijing-Kashi (c) Beijing-Sanya

Fig.4.Time synchronization error betweentwo stations caused by SinoSat-1 satellite motion

Table 1 shows the maximum values of time synchronization error between two stations when the clock biasesbetween two stations are $1 \mu \mathrm{s}, 10 \mu \mathrm{s}, 100 \mu \mathrm{s}, 1 \mathrm{~ms}$ and $10 \mathrm{~ms}$ respectively.

Table 1Time synchronization errorscaused by satellite motion

\begin{tabular}{|c|c|c|c|c|c|c|}
\hline \multirow[t]{2}{*}{$\begin{array}{l}\text { Satellite } \\
\text { name }\end{array}$} & \multirow[t]{2}{*}{ Earth station name } & \multicolumn{5}{|c|}{$\begin{array}{l}\text { The maximum error of satellite motion on time } \\
\text { synchronization } \\
\text { (unit: ps) }\end{array}$} \\
\hline & & $1 \mu \mathrm{s}$ & $10 \mu \mathrm{s}$ & $100 \mu \mathrm{s}$ & $1 \mathrm{~ms}$ & $10 \mathrm{~ms}$ \\
\hline \multirow{3}{*}{ SinoSat-1 } & Beijing-Chengdu & 7.9 & 7.9 & 8.2 & 11.3 & 42.7 \\
\hline & Beijing-Kashi & 11.1 & 11.2 & 11.5 & 14.8 & 48.3 \\
\hline & Beijing-Sanya & 15.7 & 15.8 & 16.1 & 19.2 & 50.0 \\
\hline
\end{tabular}

It can be known from Fig. 4and Table 1 that, 1) due to time out of sync between two stations, the time synchronization error for signal to reach two stations exhibits a sine wave variation with time; 
2) when the satellite is the farthest from Beijing, the time synchronization error between Beijing Station and any of other stations is the smallest; and 3) the larger the clock bias between two stations, the greater the time synchronization error for signal to reach these two stations, but when the clock biasesbetween two stations are $1 \mu \mathrm{s}, 10 \mu \mathrm{s}$ and $100 \mu$ s respectively, the time synchronization errors are almost identical, and when the clock bias is $10 \mathrm{~ms}$, the time synchronization error has the largest variation amplitude.

IGSO105 satellite

The orbital data of an IGSO satellite with a dip angle of $105^{\circ}$ on one day were selected to calculate the variation with time in distance from the satellite to Beijing, as shown in Fig. 5. Fig.6, the variations with time in time synchronization error between Beijing and Chengdu, between Beijing and Kashi and between Beijing and Sanya, respectively.

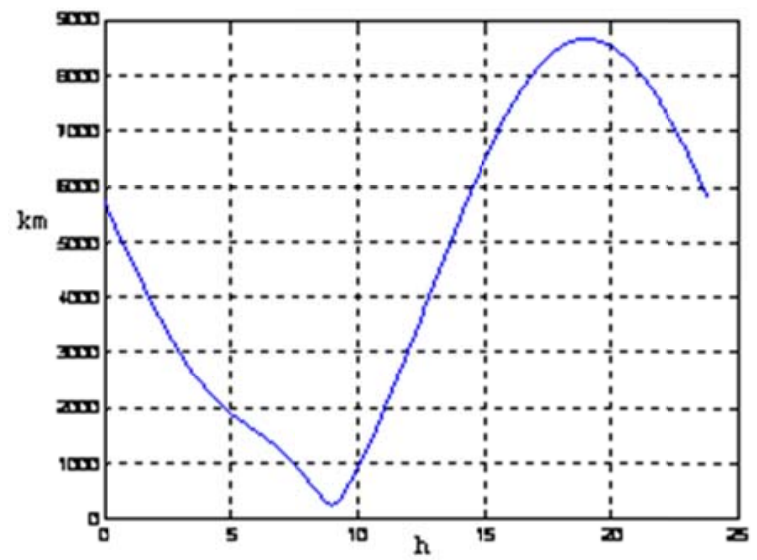

Fig.5.Variation in distance from Beijing to the IGSO105 satellite

As can be seen from Fig. 5, the variation in distance from Beijing to the IGSO105 satellite within one day exhibits an irregular graph and its range is not more than $2,466.9 \mathrm{~km}$.

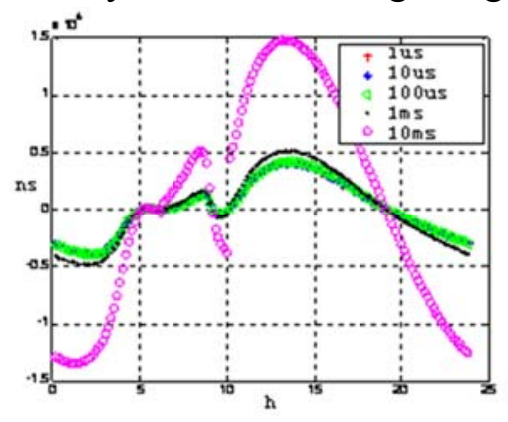

(a) Beijing-Chengdu
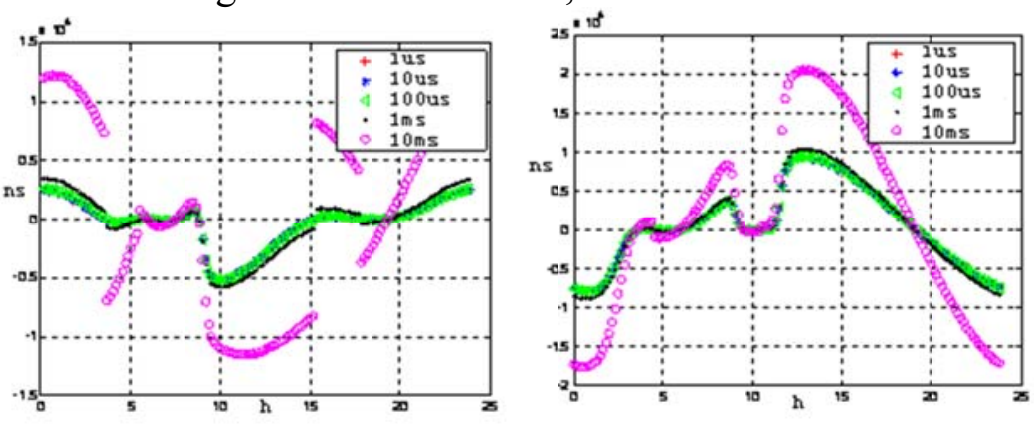

(b)Beijing-Kashi (c) Beijing-Sanya

Fig.6.Time synchronization error betweentwo stations caused by IGSO105 satellite motion

Table 2 shows the maximum values of time synchronization error between two stations when the clock biases between two stations are $1 \mu \mathrm{s}, 10 \mu \mathrm{s}, 100 \mu \mathrm{s}, 1 \mathrm{~ms}$ and $10 \mathrm{~ms}$, respectively. 
Table 2Time synchronization errorscaused by satellite motion

\begin{tabular}{|c|c|c|c|c|c|c|}
\hline \multirow{2}{*}{$\begin{array}{c}\text { Satellite } \\
\text { name }\end{array}$} & \multirow{2}{*}{ Earth station name } & \multicolumn{5}{|c|}{$\begin{array}{c}\text { The maximum error of satellite motion on time } \\
\text { synchronization } \\
\text { (unit: ps) }\end{array}$} \\
\cline { 3 - 7 } & & $1 \mu \mathrm{s}$ & $10 \mu \mathrm{s}$ & $100 \mu \mathrm{s}$ & $1 \mathrm{~ms}$ & $10 \mathrm{~ms}$ \\
\hline \multirow{3}{*}{ SinoSat-1 } & Beijing-Chengdu & 4.1 & 4.1 & 4.2 & 5.2 & 14.8 \\
\cline { 2 - 7 } & Beijing-Kashi & 2.5 & 2.5 & 2.6 & 3.5 & 12.2 \\
\cline { 2 - 7 } & Beijing-Sanya & 9.3 & 9.3 & 9.4 & 10.4 & 20.5 \\
\hline
\end{tabular}

It can be known from Fig. 6 and Table 2 that, 1) the time synchronization error for signal to reach two stations exhibits an irregular graph variation with time; 2) when the satellite is the farthest from Beijing, the time synchronization error between Beijing Station and any of other stations is the smallest; and 3) when the clock bias between two stations is $10 \mathrm{~ms}$, the time synchronization error for signal to reach two stations has the largest variation amplitude on the order of nanoseconds; it is obvious that the time error is larger than that caused by a GEO satellite; when the clock biases between two stations are $1 \mu \mathrm{s}, 10 \mu \mathrm{s}$ and $100 \mu \mathrm{s}$, respectively, the time synchronization errors for signal to reach two stations are almost identical.

\section{Conclusions}

In this paper, the influence of satellite motion on the precision of two-way time synchronization was analyzed. The influence of the synchronization error between base stations on time synchronization error for two stations to receive signal was studied using the motion characteristics of GEO and IGSO satellites.

In respect of satellite, the larger the variation in distance from Beijing Station to the satellite caused by satellite motion, the greater the time delay difference between Beijing Station and any of other stations. Due to large variation amplitude of IGSO satellite motion, the variation in time synchronization error for two stations to receive signal is relatively large, with its maximum not more than $20 \mathrm{~ns}$. The influence of GEO satellite motion on inter-station time synchronization error is smaller than that of IGSO satellite motion on inter-station time synchronization error, and is on the order of picoseconds.

In respect of clock bias, the larger the clock bias, the greater the inter-station time synchronization error; when the clock bias between two station is $10 \mathrm{~ms}$, the influence of satellite motion on the time synchronization error between the base stations is over $10 \mathrm{~ns}$. However, the inter-station time synchronization error can generally be controlled within $1 \mathrm{~ms}$. For a GEO satellite, its motion amplitude is relatively small, so the variation in time synchronization error for two stations to receive signal is also relatively small; when the clock bias between two station is within $1 \mathrm{~ms}$, the inter-station time synchronization error is not more than $20 \mathrm{ps}$.

In summary, if a GEO satellite is selected as a transfer satellite, and when the clock bias between base stations is maintained within $1 \mathrm{~ms}$, the time synchronization error between base stations caused by satellite motion can be on the order of picoseconds; at this moment, the influence of satellite motion on two-way time comparison can be neglected. If a satellite with faster motion is used, it needs to consider correction of satellite motion.

\section{Acknowledgment}

We thank Dr. Xiaohui Li for the help on this article. 


\section{References}

[1] D.Kirchner, "Two-Way Time Transfer via Communication Satellites," Proc. IEEE, vol. 79 (7), pp. 983-990, 1991.

[2] D. Kirchner, "Two-Way Satellite Time and Frequency Transfer (TWSTFT): Principle, Implementation, and Current Performance," Rev. of Radio Science, Oxford University Press, pp. 27-44, 1999.

[3] L. A. Breakiron, A. L. Smith, B. C. Fonville, E. Powers, and D. M. Matsakis, "The Accuracy of Two-Way Satellite Time Transfer Calibrations," Proc. 36th Precise Time and Time Interval (PTTI) Systems and Applications Meeting, December 2004, Washington, DC, USA, pp. 139-148, 2005.

[4] W. Schafer, A. Pawlitzki. New Trands in Two-Way Time and Frequency Transfer via Satellite[C]. 31st Annual Precise Tme and Time Interval(PTTI) Meeting, 1999, P505-514.

[5] Liu, L., and Han, C.H. Analysis of two-way satellite time comparison and its error, Progress in Astronomy, Vol. 22, No. 3, 2004. 